\title{
A novel positive feedback loop involving FASN/ p-ERK1/2/5-LOX/LTB4/FASN sustains high growth of breast cancer cells
}

\author{
Nan HU1, \#, Yu LI ${ }^{1, \#}$, Yu ZHAO ${ }^{1}$, Qi WANG ${ }^{2}$, Jia-cong YOU ${ }^{1}$, Xiao-dong ZHANG ${ }^{2, ~ * ~, ~ L i-h o n g ~ Y E ~}{ }^{1, *}$ \\ ${ }^{1}$ Department of Biochemistry, the Key Laboratory of Bioactive Materials, Ministry of Education; ${ }^{2}$ Department of Cancer Research, Key \\ Laboratory of Molecular Microbiology and Technology of Ministry of Education, Institute for Molecular Biology and Biochemistry, Col- \\ lege of Life Sciences, Nankai University, Tianjin 300071, China
}

\begin{abstract}
Aim: To investigate the endogenous signaling pathways associated with high proliferation potential of breast cancer cells. Methods: Breast cancer cell lines LM-MCF-7 and MCF-7 with high and low proliferation capability were used. The promoter activity of fatty acid synthase (FASN) was examined using luciferase reporter gene assay. The expression level of FASN mRNA was measured using RT-PCR and real time PCR, respectively. The level of leukotriene B4 (LTB4) was determined with ELISA. The expression levels of 5-lipoxygenase (5-LOX) was analyzed using RT-PCR and Western blot, respectively. 5-Bromo-20-deoxyuridine (BrdU) incorporation assay was used to study the proliferation of LM-MCF-7 and MCF-7 cells.

Results: The promoter activity of FASN was significantly higher in LM-MCF-7 cells than MCF-7 cells. Treatment of LM-MCF-7 cells with ERK1/2 inhibitor PD98059 (30-50 $\mathrm{mol} / \mathrm{L})$ or LOX inhibitor NDGA (25 $\mu \mathrm{mol} / \mathrm{L})$ abolished the activation of FASN. Moreover, treatment of LM-MCF-7 cells with the specific 5-LOX inhibitor MK-886 (20-40 $\mu \mathrm{mol} / \mathrm{L})$ or 5-LOX siRNA (50-100 nmol/L) decreased the promoter activity of FASN. The level of LTB4, the final metabolite produced by 5-LOX, was significantly higher in LM-MCF-7 cells than MCF-7 cells. Administration of exogenous LTB4 (1-10 nmol/L) was able to stimulate the promoter activity of FASN in MCF-7 cells. Treatment of LMMCF-7 cells with the FASN inhibitor cerulenin (10 $\mathrm{mmol} / \mathrm{L})$ reduced all the levels of $\mathrm{p}$-ERK1/2, 5-LOX, and LTB4. Treatment of LM-MCF-7 cells with cerulenin, PD98059, or MK-886 abolished the proliferation. Administration of exogenous LTB4 (10 nmol/L) significantly increased BrdU incorporation in MCF-7cells.

Conclusion: These results suggest a novel positive feedback loop involving FASN/p-ERK1/2/5-LOX/LTB4/FASN contributes to the sustaining growth of breast cancer LM-MCF-7 cells.
\end{abstract}

Keywords: breast cancer; extracellular signal-regulated kinase; fatty acid synthase; 5-lipoxygenase; leukotriene B4; cell proliferation

Acta Pharmacologica Sinica (2011) 32: 921-929; doi: 10.1038/aps.2011.40; published online 6 Jun 2011

\section{Introduction}

Monod and Jacob reported that the feedback loops were present in eukaryotic signal transduction system in $1961^{[1]}$. In the network of signaling transduction, the positive feedback loop regulation plays important roles in determining the progressive nature of malignant cancer cells including cell proliferation $^{[2-5]}$. Extracellular signal-regulated kinase 1/2 (ERK1/2), a major cellular proliferation signaling pathway, is involved in many positive feedback loops. Previously, we reported a positive feedback between the phosphorylated

\footnotetext{
\# These two authors contributed equally to this work.

* To whom correspondence should be addressed.

E-mail yelihong@nankai.edu.cn (Li-hong YE); zhangxd@nankai.edu.cn (Xiao-dong ZHANG)

Received 2011-02-14 Accepted 2011-03-29
}

ERK1/2 (p-ERK1/2) and cyclooxygenases (COX)/lipoxygenases $(\mathrm{LOX})^{[6]}$. Kim et al ${ }^{[7]}$ discovered a positive feedback loop between Wnt and ERK pathways. It has also been reported that there is a regulatory loop between fatty acid synthase (FASN) and ERK pathway dependent on the HER1/HER2 ${ }^{[8]}$. Cheng et $a l^{[9]}$ identified a positive feedback loop in which Ras signaling promoted CD44v6 splicing, and CD44v6 then sustained late Ras signaling. Moreover, ERK occurs crosstalk with other pathways, including activated stress activated protein kinase (SAPK)-p38 ${ }^{[10]}$, phosphatidylinositol 3-kinase $(\mathrm{PI} 3 \mathrm{~K})^{[11]}, \mathrm{Wnt}^{[12]}$ and myosin light-chain kinase $(\mathrm{MLCK})^{[13]}$. Thus, p-ERK1/2 may serve as a key hub in the network.

FASN is a $250-270 \mathrm{kDa}$ multifunctional, homodimeric enzyme responsible for energy storage by converting excess carbohydrate to fatty acids that are then sterified to store triacylglycerols ${ }^{[14]}$. Under normal physiological conditions, 
expression of FASN is tightly regulated in breast tissues, whereas deregulation of FASN occurs in cancer cells contributing to exacerbated lipogenesis required for highly proliferating cancer cell population ${ }^{[15]}$. The pharmacological and RNAi mediated inhibition of FASN leads to growth inhibition and cell apoptosis in several experimental models, suggesting that FASN may be a promising therapeutic target for cancer therapy ${ }^{[16]}$. Therefore, we are interested in the intracellular growth signaling pathways that are associated with FASN in breast cancer cells.

The contribution of arachidonic acid metabolites to cancer progression is becoming the focus of intense research, with the involvement of both COX and LOX pathways ${ }^{[17]}$. The LOX pathway has different isoenzymes, including 5-LOX, which converts arachidonic acid to hydroxyeicosatetraenoic acids or leukotrienes ${ }^{[18]}$. 5-LOX is often overexpressed in multiple tumor types and is involved in proliferation, survival, and apoptosis of cancer cells ${ }^{[19]}$. Leukotriene B4 (LTB4), the final metabolite in the 5-LOX pathway, is able to enhance proliferation, increase survival and suppress the apoptosis of human cells. Blockade of the LTB4-signaling pathway induced apoptosis via the inhibition of ERK1/2 activation in colon cancer cells ${ }^{[20]}$. However, the mechanisms that 5-LOX/LTB4 promotes breast cancer cell growth remain unclear.

Previously, our laboratory established a metastatic subclone from the MCF-7 breast cancer cell line, called LM-MCF-7, which was derived from a lung metastasis of a severe combined immunodeficient (SCID) mouse ${ }^{[21]}$. In vivo and in vitro experiments showed that LM-MCF-7 had high malignant phenotype in cell proliferation and migration ${ }^{[22]}$. The two cell lines, having the similar genetic background, provide excellent parallel models for investigating the molecular mechanisms underlying the sustained proliferation of breast cancer cells.

In the present study, we investigate the signal transduction pathway that maintains the growth of breast cancer cells using the parallel MCF-7/LM-MCF-7 breast cancer cell lines. We report a novel positive cascade loop in the network. Our data show that p-ERK1/2 is one of key hubs in the network. This finding provides new insight into the mechanisms of network regulation in breast cancer cells.

\section{Materials and methods Cell culture}

MCF-7 and LM-MCF-7 cells were cultured in RPMI-1640 (Gibco, USA) medium supplemented with $10 \%$ fetal calf serum (Gibco, USA), $100 \mathrm{U} / \mathrm{mL}$ penicillin, $100 \mathrm{mg} / \mathrm{mL}$ streptomycin and $1 \%$ glutamine. Cultures were incubated at $37^{\circ} \mathrm{C}$ in a humidified atmosphere with $5 \% \mathrm{CO}_{2}$.

\section{Reagents}

MK-886 (an inhibitor of 5-LOX), NDGA (an inhibitor of LOX), indomethacin (Indo, an inhibitor of COX), PD98059 (a p42/44 MAPK inhibitor), SKF525A (a CYP450 inhibitor) and LTB4 were purchased from Sigma-Aldrich (USA). Pertussis toxin (PTX, an inhibitor of a Gi/o protein) was purchased from List Biological Laboratories Inc (USA). Cerulenin (an inhibitor of FASN) was purchased from Fermentek Ltd (Israel). The enzyme immunoassay kit for measurement of LTB4 was purchased from Adlitteram Diagnostic Laboratories (USA).

\section{Small interfering RNA and plasmids transfection}

The reporter construct, pFASN-WT-Luc, containing the promoter of FASN was kindly provided by Dr Qiang LIU (University of Saskatchewan, Canada). The small siRNAs (siRNAs) targeting human 5-LOX mRNA (NM_000698, 315 to 335) and human FASN mRNA (NM_004104.4, 1210 to 1231) and control siRNA were designed and synthesized by RiboBio (Guangzhou, China). The transfection with RNAi reagents and different dose of plasmids were performed using Lipofectamine 2000 (Invitrogen, USA) according to the manufacturer's protocol, respectively.

\section{Treatments of tumor cells}

Cells were cultured in 24-well plates for $24 \mathrm{~h}$, and then were recultured in serum-free medium for $12 \mathrm{~h}$. In brief, LM-MCF-7 cells were treated with PTX $(30 \mathrm{ng} / \mathrm{mL}$ or $50 \mathrm{ng} / \mathrm{mL})$, Indo ( $25 \mu \mathrm{mol} / \mathrm{L})$, PD98059 (30, $50 \mu \mathrm{mol} / \mathrm{L})$, NDGA $(25 \mu \mathrm{mol} / \mathrm{L})$, SKF 525A (a CYP450 inhibitor, $50 \mathrm{\mu mol} / \mathrm{L}$ ) and AG112 (20, 40 $\mathrm{mmol} / \mathrm{L}$ ) for $4 \mathrm{~h}$, respectively. In addition, MCF-7 cells were cultured for $48 \mathrm{~h}$, followed by treatment with $50 \%$ or $100 \%$ conditioned media from LM-MCF-7 and the LM-MCF-7 cells treated with NDGA $(25 \mu \mathrm{mol} / \mathrm{L})$ for $24 \mathrm{~h}$. MCF-7 cells were treated with LTB4 $(0.1$ or $10 \mathrm{nmol} / \mathrm{L})$ for different period of time. MCF-7 cells were treated with LTB4 $10 \mathrm{nmol} / \mathrm{L}$, followed by treatment with MK-886 $(5,10$, or $20 \mu \mathrm{mol} / \mathrm{L})$ for $6 \mathrm{~h}$. LM-MCF-7 cells were treated with cerulenin $(2.5,5$, or $10 \mu \mathrm{mol} / \mathrm{L}$ ) for $12 \mathrm{~h}$. The treated cells were examined by using luciferase reporter gene assay, reverse transcription polymerase chain reaction (RT-PCR), immunoblot analysis and enzyme-linked immunosorbent (ELISA), respectively, as described below.

\section{RNA isolation, RT-PCR and quantitative real-time PCR}

Total RNA isolation, RT-PCR and quantitative real-time PCR were performed as previously described ${ }^{[23]}$. Briefly, total RNA of cells was isolated using TRIzol reagent (Invitrogen, USA) according to the manufacturer's instructions. First-strand cDNA was synthesized with PrimeScript reverse transcriptase (TaKaRa Bio, China) and oligo (dT). After reverse transcription reaction, PCR and real-time PCR were performed by an ABI PRISM 7000 sequence detection system according to the manufacturer's instructions using double stranded DNA specific flurophore SYBR Green (Promega, USA). We included specific primers for FASN (forward primer, 5'-GGT CTT GAG AGA TGG CTT GC-3' and reverse primer, 5'-AAT TGG CAA AGC CGT AGT TG-3'), 5-LOX (forward primer, 5'-CCC GGG GCA TGG AGA GCA-3' and reverse primer, 5'-GCG GTC GGG CAG CGT GTC-3'). As a control, GAPDH was amplified with specific primers (forward primer, 5 '-CAT CAC CAT CTT CCA GGA GCG-3' and reverse primer, 5'-TGA CCT TGC CCA CAG CCT TG-3'). The conditions of PCR were as follows: $94^{\circ} \mathrm{C}$ for $10 \mathrm{~min}$, followed by 35 cycles of amplifications 
including $94{ }^{\circ} \mathrm{C}$ for $30 \mathrm{~s}, 52^{\circ} \mathrm{C}$ for $45 \mathrm{~s}$, and $72{ }^{\circ} \mathrm{C}$ for $45 \mathrm{~s}$, and a final extension at $72{ }^{\circ} \mathrm{C}$ for $10 \mathrm{~min}$. The conditions of realtime PCR were as follows: $95{ }^{\circ} \mathrm{C}$ for $10 \mathrm{~s}, 95^{\circ} \mathrm{C}$ for $5 \mathrm{~s}, 53^{\circ} \mathrm{C}$ annealing for $1 \mathrm{~min}, 72{ }^{\circ} \mathrm{C}$ for $15 \mathrm{~s}$, followed by 45 cycles. There is no nonspecific amplification determined by dissolved curves. There are three samples in each group. The real-time PCR results were reported as the fold induction of relative light units for the treatment over vehicle after normalization to GAPDH expression.

\section{Luciferase reporter gene assay}

Treated MCF-7 and LM-MCF-7 cells grown in 24-well plates were cotransfected with $0.3 \mu \mathrm{g}$ of a plasmid encoding firefly luciferase under the control of wild-type FASN promoter, and $0.05 \mu \mathrm{g}$ of pRL-SV40 encoding Renilla luciferase (rLuc) (Promega, USA). Reporter luciferase assay was performed $48 \mathrm{~h}$ after transfection with Dual Luciferase Assay reagents (Promega, USA) using a TD 20/20 luminometer (Turner Designs). The luciferase readings of each sample were normalized against the rLuc levels. All of the data shown in this study were obtained from three independent experiments.

\section{Western blot analysis}

Cells were washed with cold phosphate-buffered saline (PBS) for 3 times and lysed in RIPA cell lysis buffer $(10 \mathrm{mmol} / \mathrm{L}$ HEPES pH 7.4, $0.15 \mathrm{~mol} / \mathrm{L} \mathrm{NaCl}, 1 \mathrm{mmol} / \mathrm{L} \mathrm{MgCl}_{2}, 1 \mathrm{mmol} / \mathrm{L}$ $\mathrm{CaCl}_{2}, 1 \mathrm{mmol} / \mathrm{L}$ dithiothreitol, $0.1 \%$ sodium dodecylsulfate, $0.1 \% \mathrm{NP}-40$, and $20 \mu \mathrm{g} / \mathrm{mL}$ leupeptin). Protein concentrations were estimated using Bradford reagent (Bio-Rad, USA). Equal amount of total protein was loaded for immunoblotting. Following SDS-PAGE, resolved proteins were electrotransfered on PVDF membrane (Millipore, USA). The membrane was blocked overnight in TBS containing $0.1 \%$ Tween-20 (TBST) and 5\% skim milk. The membrane was then probed with primary antibody in TBST for $2 \mathrm{~h}$ at room temperature or overnight at $4{ }^{\circ} \mathrm{C}$, followed by three 15 min TBST washes at room temperature. Incubation with the secondary antibody was done for $1 \mathrm{~h}$ and three $10 \mathrm{~min}$ TBST washes were given prior to chemiluminiscence detection using ECL substrate (Amersham Biosciences, UK). The primary antibodies for Western blot followed a dilution below mouse anti-p-ERK1/2 monoclonal antibody (1:1000 dilution, Cell Signaling Technology, Danvers, MA, USA), rabbit anti-5-LOX monoclonal antibody (1:500 dilution, Santa Cruz Biotechnology), mouse anti-human $\beta$-actin monoclonal antibody (1:20 000 dilution, Sigma). The data were analyzed by applying Glyco Band-Scan software. All of the data shown in this study were obtained from three independent experiments.

\section{Enzyme-linked immunosorbent assay (ELISA)}

The amount of LTB4, a metabolite of 5-LOX, was determined by ELISA assay according to the instructions provided by the manufacturer. The concentration of LTB4 was normalized to total protein. The concentrations of LTB4 in these extracts were determined using a protein assay method (Bio-Rad).

\section{BrdU incorporation assay}

The detailed 5-Bromo-20-deoxyuridine (BrdU) incorporation assay procedure was described as previously ${ }^{[13]}$. In brief, LMMCF-7 and MCF-7 cells were seeded in 6-well culture plate and were grown overnight prior to treatment. LM-MCF-7 cells were treated with $2.5,5$, and $10 \mu \mathrm{mol} / \mathrm{L}$ cerulenin for $8 \mathrm{~h}$, or with 10 and $20 \mu \mathrm{mol} / \mathrm{L}$ MK-886 for $8 \mathrm{~h}$, respectively. MCF-7 cells were treated with 1 and $10 \mathrm{nmol} / \mathrm{L} \mathrm{LTB} 4$ for $8 \mathrm{~h}$. All groups $(n=3$, in every group) were incubated with fresh medium containing $10 \mu \mathrm{mol} / \mathrm{L} \mathrm{BrdU}$ (Sigma) for $4 \mathrm{~h}$ prior to immunofluorescence staining with mouse anti-BrdU antibody. The cells were fixed for 15 min with $4 \%$ paraformaldehyde in PBS. After $1 \mathrm{~h}$ incubation with PBS containing $2 \mathrm{~mol} / \mathrm{L} \mathrm{HCl}$ to denature DNA, cover slips were washed 3 times with $0.5 \%$ bovine serum albumin (BSA) and 0.5\% Tween 20 in PBS, and incubated overnight $\left(4^{\circ} \mathrm{C}\right)$ with a mouse anti-BrdU antibody (NeoMarkers, Fremont, CA, USA) at 1:300 dilution. Reactions were developed using fluorescein isothiocyanate (FITC)conjugated goat anti-mouse IgG (Dako, Glostrup, Denmark) at 1:100 dilution for BrdU staining. The BrdU labeling index was assessed by point counting through a Nikon TE200 inverted microscope (Nikon, Tokyo, Japan) using a $40 \times$ objective lens. About 700-800 nuclei were counted in 6-8 representative fields. The labeling index was expressed as the number of positively labeled nuclei/total number of nuclei. Propidium iodine (PI) (Sigma) (50 $\mu \mathrm{g} / \mathrm{mL})$ was used to stain nuclei as the control to all cells in each group.

\section{Statistical analysis}

Statistical analysis was performed using SigmaPlot 2001 (Systat Software Inc, Richmond, CA, USA http://www.systat. com). Statistical significance was assessed by comparing the mean values $( \pm S D)$ using a Student's $t$ test or $\chi^{2}$ test.

\section{Results}

\section{p-ERK1/2 and LOX are involved in the upregulation of FASN}

It has been reported that FASN is associated with the progression of many kinds of tumor and it is primarily regulated at the transcriptional level. Then, we examined the promoter activities of FASN in MCF-7 and LM-MCF-7 cells with different metastatic ability. Our data showed that the promoter activity of FASN was much higher in LM-MCF-7 cells with high proliferation capability relative to MCF-7 cells using pFASN-WT-Luc, a FASN promoter-luciferase reporter (Figure $1 \mathrm{~A}, P<0.05$ vs MCF-7 cells, Student's $t$ test). To identify the signaling pathways involved in FASN, we undertook a screen of several cellular proliferation signaling pathways by treatment with different inhibitors in LM-MCF-7 cells. Our data showed that $30 \mu \mathrm{mol} / \mathrm{L}$ and $50 \mu \mathrm{mol} / \mathrm{L}$ PD98059 (a p42/44 MAPK inhibitor) and $25 \mu \mathrm{mol} / \mathrm{L}$ NDGA (a LOX inhibitor) significantly decreased the promoter activity of FASN. However, no modulation was observed after the addition of $30 \mathrm{ng} / \mathrm{mL}$ and $50 \mathrm{ng} / \mathrm{mL}$ PTX (a Gi/o protein inhibitor), $20 \mu \mathrm{mol} / \mathrm{L}$ and $40 \mu \mathrm{mol} / \mathrm{L}$ AG112 (a specific EGFR kinase inhibitor), 25 $\mu \mathrm{mol} / \mathrm{L}$ indomethacin (Indo, a COX inhibitor) and $50 \mu \mathrm{mol} / \mathrm{L}$ 

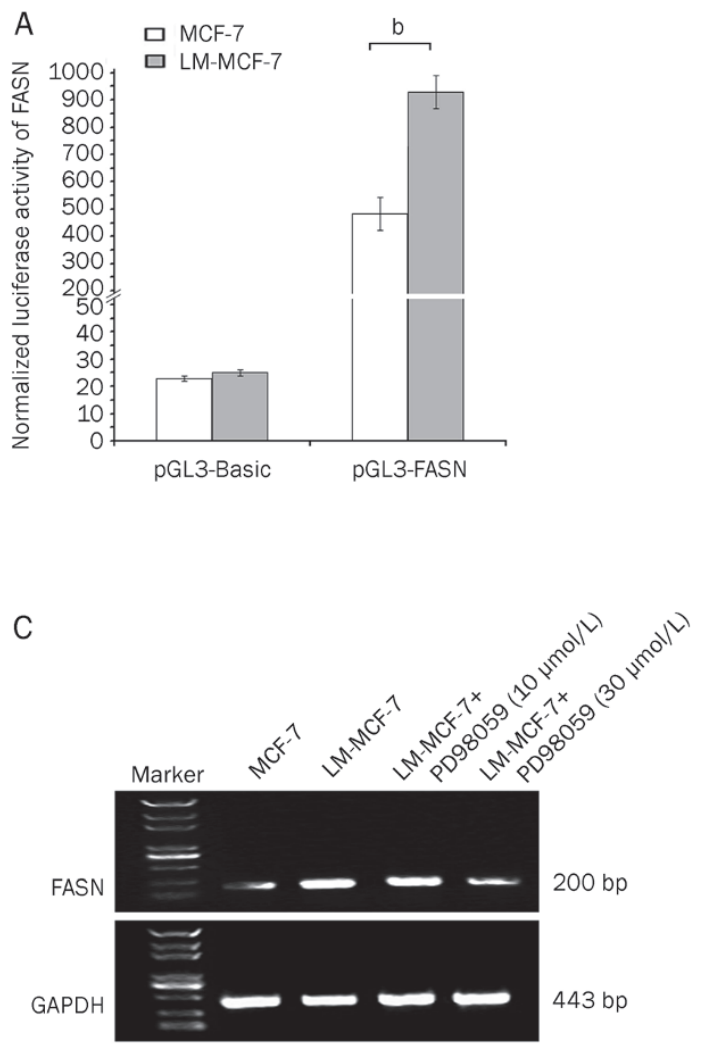
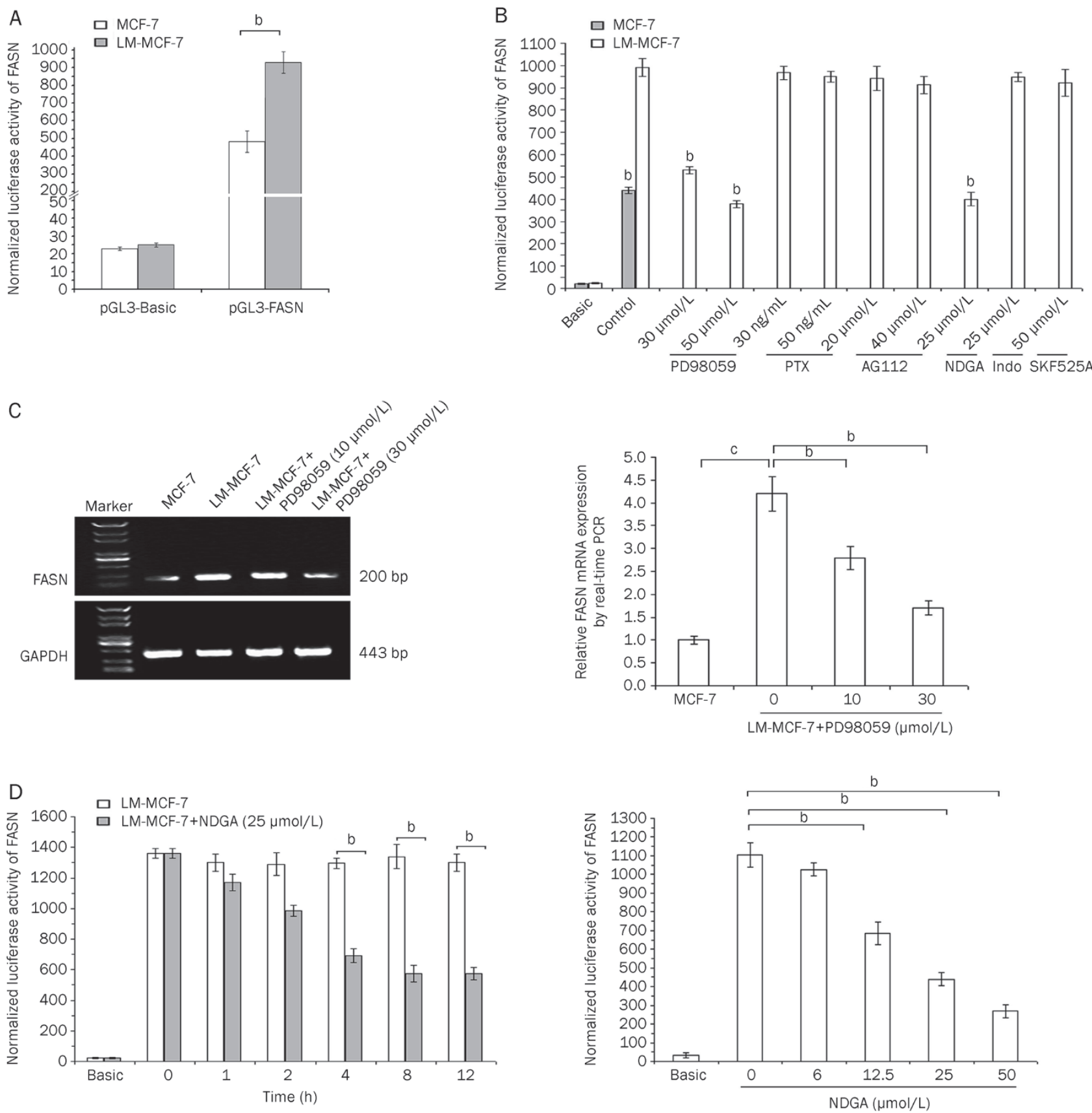

Figure 1. $\mathrm{p}$-ERK1/2 and LOX are responsible for the upregulation of FASN. (A) The promoter activity of FASN was examined by luciferase reporter gene assay in MCF-7 and LM-MCF-7 cells ( ${ }^{b} P<0.05$ vs MCF-7 cells, Student's $t$ test). (B) The promoter activity of FASN was examined by luciferase reporter gene assay in LM-MCF-7 cells treated with PD98059 (a p42/44 MAPK inhibitor), PTX (a Gi/o protein inhibitor), AG112 (a specific EGFR kinase inhibitor), NDGA (an inhibitor of LOX), Indo (indomethacin, a COX inhibitor), and SKF525A (a CYP450 inhibitor), respectively ( ${ }^{\mathrm{b} P}<0.05$ vs LM-MCF-7 cells, Student's $t$ test). (C) The expression of FASN mRNA was measured by RT-PCR and real time PCR, respectively, after treatment with PD98059 $\left({ }^{b} P<0.05\right.$; ${ }^{\circ} P<0.01$ vs LM-MCF-7 cells, Student's $t$ test). (D) The promoter activity of FASN was examined by luciferase reporter gene assay in LM-MCF-7 cells treated with NDGA ( ${ }^{b} P<0.05$ vs LM-MCF-7 cells, Student's $t$ test). All data are obtained from at least three independent experiments. Bars indicate the standard deviations.

SKF525A (a CYP450 inhibitor), respectively (Figure 1B, $P<0.05$ vs LM-MCF-7 cells, Student's $t$ test). Furthermore, RT-PCR and quantitative real time PCR showed that PD98059 reduced mRNA level of FASN in a dose-dependent manner in LMMCF-7 cells (Figure 1C, $P<0.05 ; P<0.01$ vs LM-MCF-7 cells,
Student's $t$ test). We also found that NDGA reduced the promoter activity of FASN in a dose- and time-dependent manner (Figure 1D, $P<0.05$ vs LM-MCF-7 cells, Student's $t$ test). Thus, these data suggest that $\mathrm{p}$-ERK1/2 and LOX are upstream activators of FASN. 
p-ERK1/2 activates 5-LOX

Our previous finding showed that PD98059 treatment led to a decrease of 5-LOX expression in breast cancer cells ${ }^{[6]}$. Here, we confirmed the results in the two cell lines as determined by RT-PCR and Western blot analysis, respectively (data not shown).

\section{5-LOX/LTB4 activates FASN}

Next, we investigated whether 5-LOX was involved in the upregulation of FASN. We treated LM-MCF-7 cells with increasing concentration of MK-886 (a specific 5-LOX inhibitor) for $6 \mathrm{~h}$. Our finding showed that MK-886 was able to decrease the expression of FASN in a dose-dependent manner in LM-MCF-7 cells by luciferase reporter gene assays, RT-PCR and real-time PCR, respectively (Figure 2A-2B, $P<0.05 ; P<0.01$ vs LM-MCF-7 cells, Student's $t$ test). In addition, we found that siRNA targeting the mRNA of 5-LOX could significantly
A
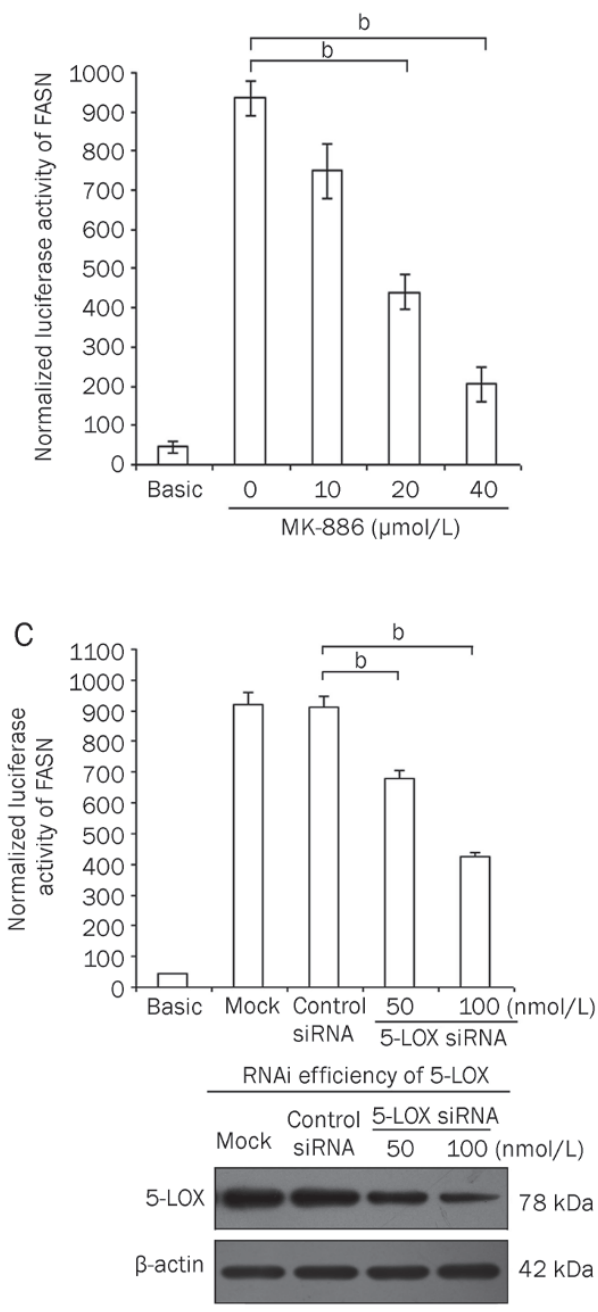

$\mathrm{E}$

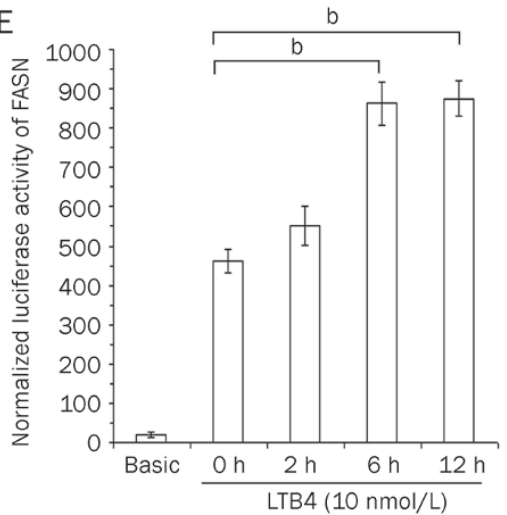

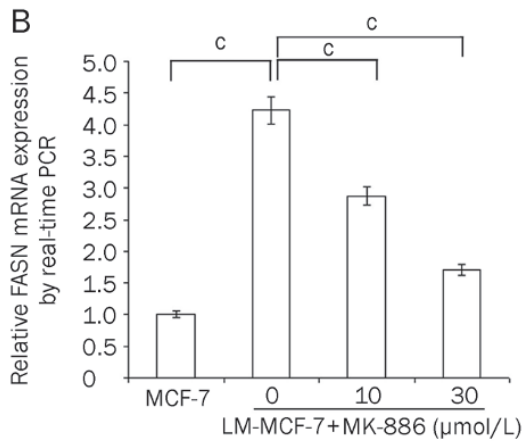
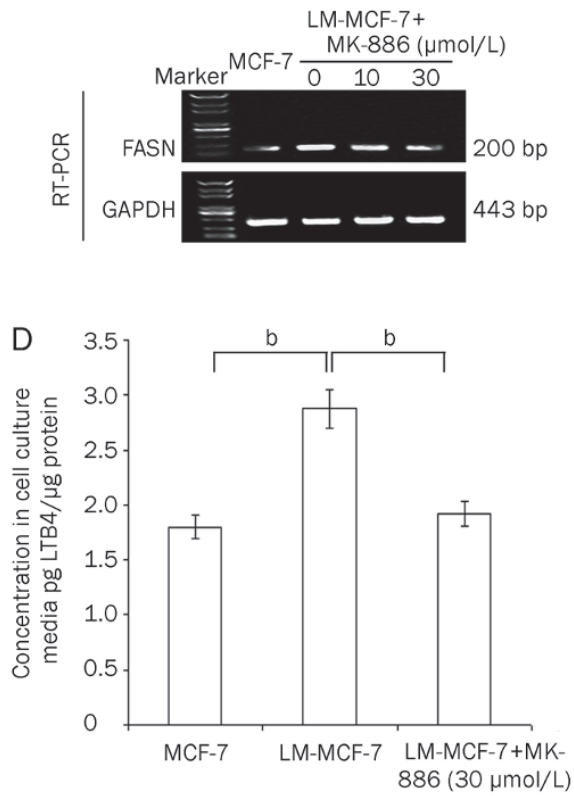

$\mathrm{F}$

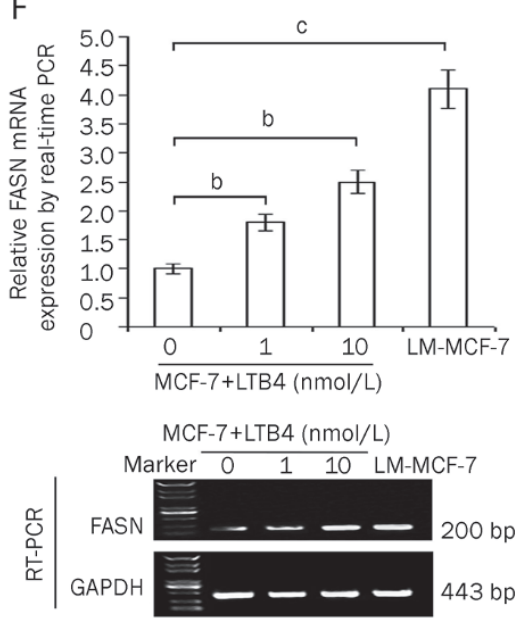

Figure 2. 5-LOX/LTB4 activates FASN. (A) The promoter activity of FASN was examined by luciferase reporter gene assay in LM-MCF-7 cells treated with MK$886\left({ }^{b} P<0.05\right.$ vs LM-MCF-7 cells, Student's $t$ test). (B) The mRNA levels of FASN was examined by RT-PCR and real time PCR in LM-MCF-7 cells treated with MK-886 $\left({ }^{\mathrm{C} P} P 0.01\right.$ vs LM-MCF-7 cells, Student's $t$ test). (C) LM-MCF-7 cells were transfected with 5-LOX siRNA (50 or $100 \mathrm{nmol} / \mathrm{L}$ ) or control siRNA for $48 \mathrm{~h}$. Then, the promoter activities of FASN were examined by luciferase reporter gene assay $\left({ }^{b} P<0.05\right.$ vs control siRNA, Student's $t$ test). In parallel, the protein levels of 5-LOX were analyzed with Western blot analysis. (D) The levels of LTB4 were determined by ELISA in the conditioned media from MCF-7, LM-MCF-7, and LM-MCF-7 cells treated with MK-886, respectively $\left({ }^{\mathrm{b} P}<0.05\right.$ vs LM-MCF- 7 cells, Student's $t$ test). (E) The promoter activity of FASN was examined by luciferase reporter gene assay in MCF-7 cells treated with exogenous LTB4 $\left({ }^{b} P<0.05\right.$ vs MCF-7 cells, Student's $t$ test). (F) The mRNA levels of FASN was examined by RT-PCR and real time PCR in MCF-7 cells treated with exogenous LTB4 $\left({ }^{b} P<0.05,{ }^{c} P<0.01\right.$ vs MCF-7 cells, Student's $t$ test). All data are obtained from at least three independent experiments. Bars indicate the standard deviations. 
reduce the promoter activity of FASN in a dose-dependent manner in LM-MCF-7 cells as well (Figure $2 \mathrm{C}, P<0.05$ vs control siRNA, Student's $t$ test). Then, we determined the amount of LTB4, the final metabolite of 5-LOX, in the conditioned media of MCF-7 and LM-MCF-7 cells. ELISA analysis showed that the levels of released LTB4 were higher in the conditioned media from LM-MCF-7 cells than that in the conditioned media from MCF-7 cells. However, this elevation could be abolished by pretreatment with MK-886 (Figure 2D, $P<0.05$ vs LM-MCF-7 cells, Student's $t$ test). Accordingly, we examined whether LTB4 was responsible for high level of FASN. The administration of exogenous LTB4 was able to enhance the promoter activity of FASN in a time-dependent manner by luciferase reporter gene assay in MCF-7 cells (Figure 2E, $P<0.05$ vs MCF-7 cells, Student's $t$ test). Meanwhile, LTB4 significantly increased the FASN expression by RT-PCR and realtime PCR in the cells (Figure 2F, $P<0.05 ; P<0.01$ vs MCF-7 cells, Student's $t$ test). 5-HETE, one of 5-LOX important products, has direct proliferating activity on a subset of cancers ${ }^{[24]}$. To demonstrate whether 5-HETE contributes to the upregulation of FASN, we examined the effect of 5-HETE on FASN promoter activity. Our data showed that the treatment with 5-HETE in MCF-7 cells failed to activate the promoter activity of FASN (data not shown). Thus, it suggests that 5-LOX/ LTB4, but not 5-HETE, is responsible for the upregulation of FASN.

\section{LTB4/FASN activates the p-ERK1/2/5-LOX in a positive feedback manner}

Given several studies revealed that the effects of LTB4 were mediated through MEK/ERK pathway ${ }^{[25-27]}$, we speculated whether LTB4 could activate p-ERK1/2/5-LOX in a positive feedback manner in breast cancer MCF-7 cells. Interestingly, Western blot analysis showed that administration of exogenous LTB4 was able to increase the expression levels of p-ERK1/2 and 5-LOX in a dose-dependent manner (Figure $3 \mathrm{~A})$. In addition, we also investigated whether FASN could activate p-ERK1/2/5-LOX/LTB4 signaling in a positive

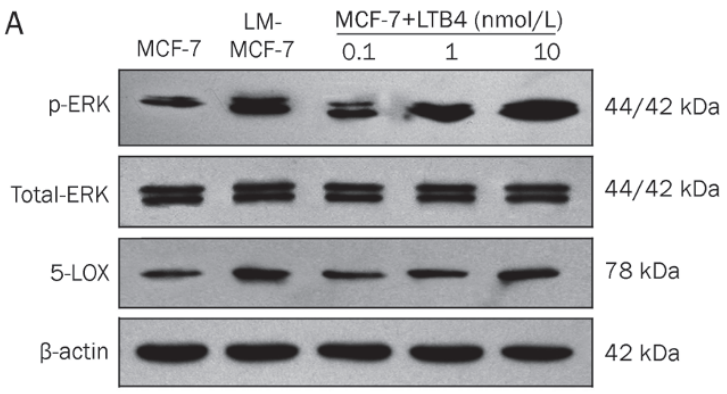

B

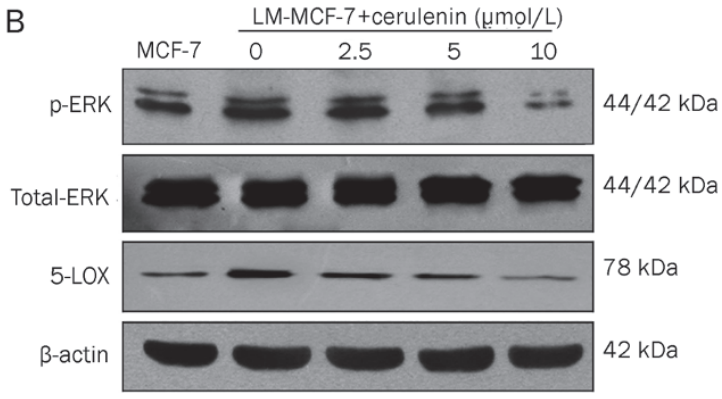

C

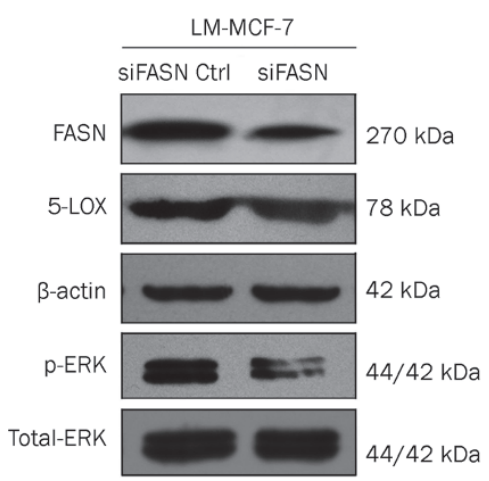

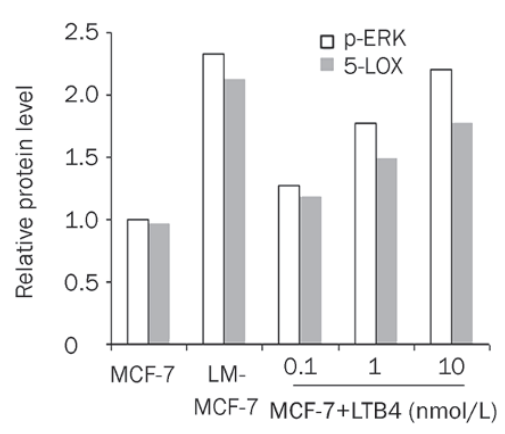
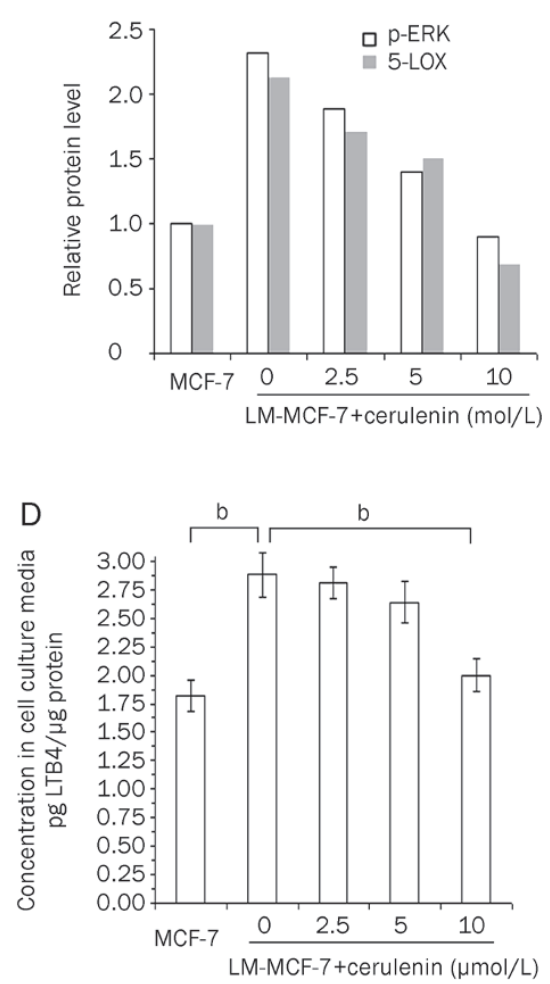

Figure 3. LTB4/FASN activates the p-ERK1/2/5-LOX in a positive feedback manner. (A) The expression levels of p-ERK1/2 and 5-LOX were examined by Western blot analysis in MCF-7 cells treated with exogenous LTB4. Histogram shows the results by applying Glyco Band-Scan software. (B) The expression levels of $p$-ERK1/2 and 5-LOX were examined by Western blot analysis in LM-MCF-7 cells treated with cerulenin, an inhibitor of FASN. Histogram shows the results by applying Glyco BandScan software. (C) The expression levels of FASN, p-ERK1/2 and 5-LOX were examined by Western blot analysis in LM-MCF-7 cells treated with siRNA targeting FASN mRNA. (D) The levels of released LTB4 were examined by ELISA assay in the conditioned media from LM-MCF-7 cells treated with cerulenin $\left({ }^{b} P<0.05\right.$ vs LM-MCF- 7 cells, Student's $t$ test). All data are obtained from at least three independent experiments. Bars indicate the standard deviations. 
feedback manner. The treatment with cerulenin, an inhibitor of FASN, could greatly decrease the levels of p-ERK1/2 and 5-LOX in a dose-dependent manner in LM-MCF-7 cells (Figure 3B). Silencing of FASN with siRNA targeting FASN mRNA could also significantly reduce the levels of $\mathrm{p}$-ERK1/2 and 5-LOX (Figure 3C). Furthermore, cerulenin decreased the levels of LTB4 in a dose-dependent manner in LM-MCF-7 cells (Figure 3D, $P<0.05$ vs LM-MCF-7 cells, Student's $t$ test), suggesting that LTB4/FASN activates the signaling of p-ERK1/2/5-LOX in a positive feedback manner.

A positive feedback loop of FASN/p-ERK1/2/5-LOX/LTB4/FASN maintains growth of breast cancer cells

Taken together, we summarize that FASN/p-ERK1/2/5LOX/LTB4/FASN results in a positive feedback loop. Then, we investigated the effect of the loop on keeping the growth of breast cancer cells by BrdU incorporation assay. Our results revealed that the treatment with cerulenin (or PD98059, MK-886) abolished the proliferation of LM-MCF-7 cells (Figure $4 \mathrm{~A}, P<0.05$ vs LM-MCF-7 cells, Student's $t$ test). The administration of exogenous LTB4 significantly increased BrdU labeling index of MCF-7 cells (Figure 4B, $P<0.05$ vs MCF-7 cells, Student's $t$ test). Thus, it suggests that the novel positive feedback loop of FASN/p-ERK1/2/5-LOX/LTB4/FASN is responsible for maintaining the growth of breast cancer cells.

\section{Discussion}

Previously, we reported that breast cancer cells grew faster in a positive feedback manner involving activated ERK and COX/LOX ${ }^{[6]}$. However, the endogenous signaling pathways associated with high proliferation potential of breast cancer cells remain unclear. Indeed, we are interested in the network regulation involving positive feedback loop manner in sustaining tumor growth. In this study, we focused on the investigation of positive feedback loop.

FASN is a complex multifunctional enzyme that plays a central role in endogenous lipogenesis in mammals ${ }^{[28,29]}$. It has been reported that the overexpression of FASN together with a high proliferative index of breast cancer cells are associated with a nine-fold increased risk of patient mortality ${ }^{[30]}$. Accordingly, we examined the level of FASN by comparing breast cancer cell lines with different proliferative capability. Our data showed that the level of FASN promoter activity was positively correlated with the high proliferative ability
A
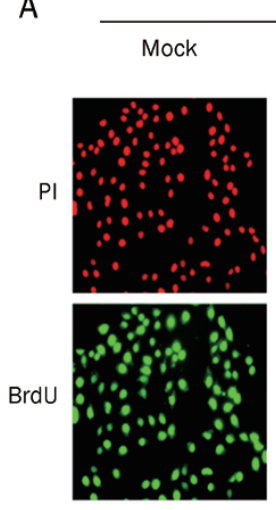

LM-MCF-7
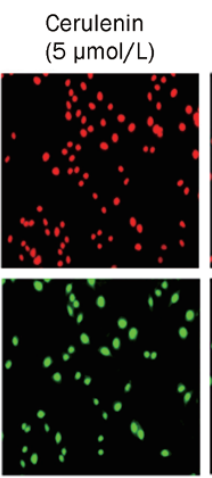

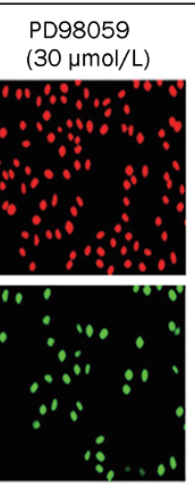

MK-886 $(30 \mu \mathrm{mol} / \mathrm{L})$
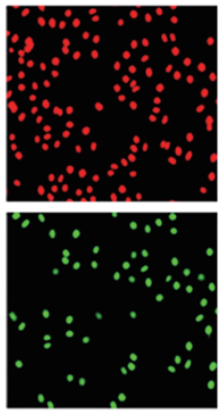

B
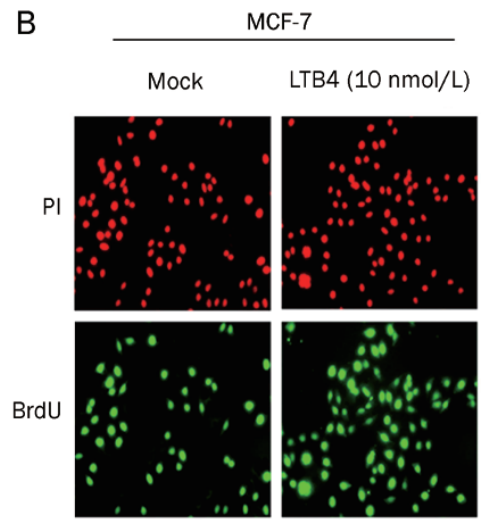
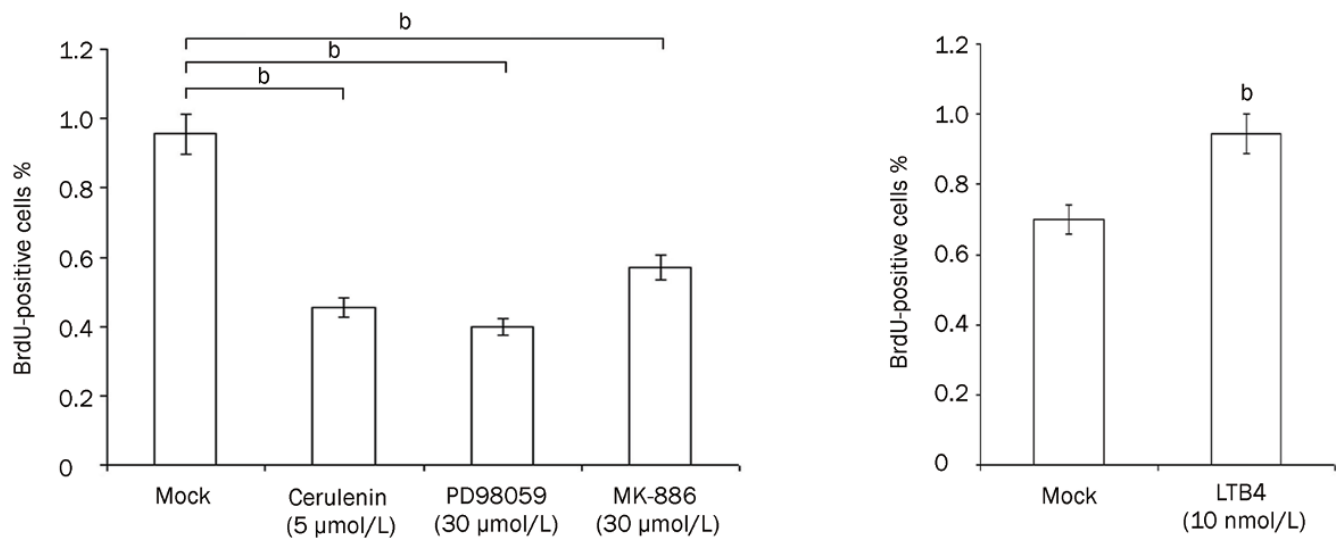

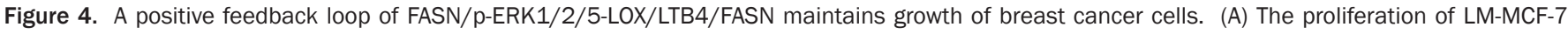

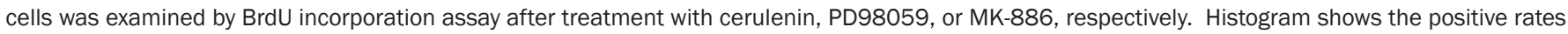

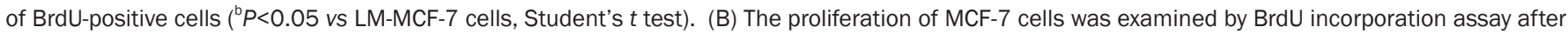

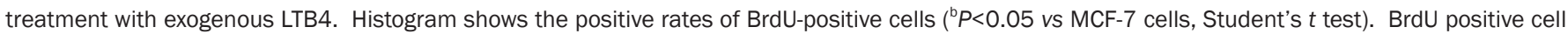

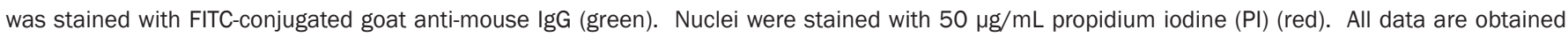
from at least three independent experiments. Bars indicate the standard deviations. 
(Figure 1A). Thus, we need to answer the upstream activators of FASN in breast cancer cells. Several studies have demonstrated the involvement of ERK1/2 pathways in regulating FASN expression in cancer cells ${ }^{[31,32]}$. It has been reported that LOX or its metabolites are able to stimulate tumor cell proliferation $^{[33]}$. In agreement, we identified that $\mathrm{p}$-ERK1/2 and LOX were upstream regulators of FASN (Figure 1B-1D).

Several studies have reported that 5-LOX is able to enhance cell proliferation and increase cell survival ${ }^{[19,34]}$. A recent study found that the expression levels of FASN and 5-LOX played important roles in mediating breast cancer formation ${ }^{[35]}$. Our previous observation showed that ERK1/2 activation increased the expression of 5-LOX in breast cancer cells ${ }^{[6]}$. In this text, we confirmed the data (data not shown). Activation of ERK1/2 and overexpression of 5-LOX are frequently observed in various human tumors ${ }^{[36,37]}$. Thus, we conclude that p-ERK1/2 activates 5-LOX in breast cancer cells. The final product of the 5-LOX pathway is LTB4, which is able to enhance tumor growth, including enhancing proliferation and suppressing apoptosis in human cancer cells ${ }^{[38]}$. Therefore, we present a hypothesis that a link between 5-LOX metabolism and FASN may contribute to sustaining high proliferation rate of breast cancer cells. Interestingly, we found that the blockade of 5-LOX with MK-886 or siRNAs was able to significantly decrease the expression level of FASN in LM-MCF-7 cells (Figure $2 \mathrm{~A}-2 \mathrm{C}$ ). Then, we examined the amount of LTB4 in the conditioned media from breast cancer cells. Our data showed that the level of LTB4 was higher in the cellular extracts from LM-MCF-7 cells relative to MCF-7 cells, which was abolished by the treatment with MK-886 (Figure 2D). Interestingly, the administration of exogenous LTB4 was able to enhance the expression of FASN (Figure 2E-2F). Moreover, we examined the effect of 5-HETE, another product of 5-LOX, on FASN. Our data showed that 5-HETE failed to activate the promoter activity of FASN in MCF-7 cells. Thus, we conclude that 5-LOX/LTB4, but not 5-HETE, is responsible for the upregulation of FASN.

Currently, feedback mechanisms have been implicated in the carcinogenesis. Our previous study showed that 5-LOX plays an important role in the activation of ERK $1 / 2^{[39]}$. Studies by Tong $e t$ al $l^{[25]}$ have shown that the growth stimulatory effect of LTB4 is mediated at least through MEK/ERK pathway in human pancreatic cancer cells. Consistently, we here report a novel positive feedback loop involving FASN/p-ERK1/2/5LOX/LTB4/FASN that is responsible for the sustained high proliferation of breast cancer cells (Figures 3, 4).

Taken together, we conclude that a novel positive feedback loop involving FASN/p-ERK1/2/5-LOX/LTB4/FASN sustains a high proliferative potential of breast cancer cells, in which p-ERK1/2 is one of key hubs in the network. Our finding provides a new insight into the mechanism involving the high growth ability of breast cancer cells.

\section{Acknowledgements}

The authors thank Dr Qiang LIU (University of Saskatchewan, Canada) for providing plasmid of pFASN-WT-Luc. This work was supported in part by National Basic Research Program of China (973 Program, № 2007CB914802, 2007CB914804, and 2009CB521702), National Natural Scientific Foundation of China (№ 81071623 and 30770826) and Tianjin Natural Scientific Foundation (№ 08JCZDJC20700).

\section{Author contribution}

Li-hong YE and Xiao-dong ZHANG designed the research; Nan HU, Yu LI, Yu ZHAO, Qi WANG, and Jia-cong YOU performed the experiments; Li-hong YE, Xiao-dong ZHANG, and Nan HU analyzed data and wrote the paper.

\section{References}

1 Monod J, Jacob F. Teleonomic mechanisms in cellular metabolism, growth, and differentiation. Cold Spring Harb Symp Quant Biol 1961; 26: 389-401.

2 Wanzel M, Russ AC, Kleine-Kohlbrecher D, Colombo E, Pelicci PG, Eilers M. A ribosomal protein L23-nucleophosmin circuit coordinates Mizl function with cell growth. Nat Cell Biol 2008; 10: 1051-61.

3 Li H, Xu LL, Masuda K, Raymundo E, McLeod DG, Dobi A, et al. A feedback loop between the androgen receptor and a NEDD4-binding protein, PMEPA1, in prostate cancer cells. J Biol Chem 2008; 283: 28988-95.

4 Wang HQ, Altomare DA, Skele KL, Poulikakos PI, Kuhajda FP, Di Cristofano A, et al. Positive feedback regulation between AKT activation and fatty acid synthase expression in ovarian carcinoma cells. Oncogene 2005; 24: 3574-82.

5 Rasola A, Fassetta M, De Bacco F, D'Alessandro L, Gramaglia D, Di Renzo MF, et al. A positive feedback loop between hepatocyte growth factor receptor and beta-catenin sustains colorectal cancer cell invasive growth. Oncogene 2007; 26: 1078-87.

6 You J, Mi D, Zhou X, Qiao L, Zhang H, Zhang X, et al. A positive feedback between activated extracellularly regulated kinase and cyclooxygenase/lipoxygenase maintains proliferation and migration of breast cancer cells. Endocrinology 2009; 150: 1607-17.

7 Kim D, Rath O, Kolch W, Cho KH. A hidden oncogenic positive feedback loop caused by crosstalk between Wnt and ERK pathways. Oncogene 2007; 26: 4571-9.

8 Vazquez-Martin A, Colomer R, Brunet J, Lupu R, Menendez JA. Overexpression of fatty acid synthase gene activates HER1/HER2 tyrosine kinase receptors in human breast epithelial cells. Cell Prolif 2008; 41: 59-85.

9 Cheng C, Yaffe MB, Sharp PA. A positive feedback loop couples Ras activation and CD44 alternative splicing. Genes Dev 2006; 20: 1715-20.

10 Estrada Y, Dong J, Ossowski L. Positive crosstalk between ERK and p38 in melanoma stimulates migration and in vivo proliferation. Pigment Cell Melanoma Res 2009; 22: 66-76.

11 Bradley EW, Ruan MM, Vrable A, Oursler MJ. Pathway crosstalk between Ras/Raf and PI3K in promotion of M-CSF-induced MEK/ERKmediated osteoclast survival. J Cell Biochem 2008; 104: 1439-51.

$12 \mathrm{Hu}$ T, Li C. Convergence between Wnt-beta-catenin and EGFR signaling in cancer. Mol Cancer 2010; 9: 236.

13 Zhou X, Liu Y, You J, Zhang H, Zhang X, Ye L. Myosin light-chain kinase contributes to the proliferation and migration of breast cancer cells through cross-talk with activated ERK1/2. Cancer Lett 2008; 270: 312-27.

14 Flavin R, Peluso S, Nguyen PL, Loda M. Fatty acid synthase as a potential therapeutic target in cancer. Future Oncol 2010; 6: 551-62.

15 Little JL, Kridel SJ. Fatty acid synthase activity in tumor cells. Subcell 
Biochem 2008; 49: 169-94.

16 Lupu R, Menendez JA. Pharmacological inhibitors of fatty acid synthase (FASN) - catalyzed endogenous fatty acid biogenesis: a new family of anti-cancer agents? Curr Pharm Biotechnol 2006; 7: 48393.

17 Maloberti PM, Duarte AB, Orlando UD, Pasqualini ME, Solano AR, Lopez-Otin $\mathrm{C}$, et al. Functional interaction between acyl-CoA synthetase 4 , lipooxygenases and cyclooxygenase-2 in the aggressive phenotype of breast cancer cells. PLoS One 2010; 5: e15540.

18 Catalano A, Procopio A. New aspects on the role of lipoxygenases in cancer progression. Histol Histopathol 2005; 20: 969-75.

19 Chen X, Sood S, Yang CS, Li N, Sun Z. Five-lipoxygenase pathway of arachidonic acid metabolism in carcino-genesis and cancer chemoprevention. Curr Cancer Drug Targets 2006; 6: 613-22.

20 Ihara A, Wada K, Yoneda M, Fujisawa N, Takahashi H, Nakajima A. Blockade of leukotriene B4 signaling pathway induces apoptosis and suppresses cell proliferation in colon cancer. J Pharmacol Sci 2007; 103: 24-32.

21 Ye LH, Wu LY, Guo W, Ma HT, Zhang XD. Screening of a sub-clone of human breast cancer cells with high metastasis potential. Zhonghua Yi Xue Za Zhi 2006; 86: 61-5.

22 Ye LH, You JC, Guo W, Xu SF, Wu LY, Qi RZ, et al. Investigation of discrepant proteins between two breast cancer cell lines with different metastatic abilities. Ai Zheng 2006; 25: 823-7.

23 Cui W, Zhang Y, Hu N, Shan C, Zhang S, Zhang W, et al. MiRNA-520b and miR-520e sensitize breast cancer cells to complement attack via directly targeting 3'UTR of CD46. Cancer Biol Ther 2010; 10: 23241.

24 Hayashi T, Nishiyama K, Shirahama T. Inhibition of 5-lipoxygenase pathway suppresses the growth of bladder cancer cells. Int J Urol 2006; 13: 1086-91.

25 Tong WG, Ding XZ, Talamonti MS, Bell RH, Adrian TE. LTB4 stimulates growth of human pancreatic cancer cells via MAPK and PI-3 kinase pathways. Biochem Biophys Res Commun 2005; 335: 949-56.

26 Sanchez-Galan E, Gomez-Hernandez A, Vidal C, Martin-Ventura JL, Blanco-Colio LM, Munoz-Garcia B, et al. Leukotriene B4 enhances the activity of nuclear factor-kappaB pathway through BLT1 and BLT2 receptors in atherosclerosis. Cardiovasc Res 2009; 81: 216-25.

27 Woo CH, You HJ, Cho SH, Eom YW, Chun JS, Yoo YJ, et al. Leukotriene $\mathrm{B}(4)$ stimulates Rac-ERK cascade to generate reactive oxygen species that mediates chemotaxis. J Biol Chem 2002; 277: 8572-8.

28 Kridel SJ, Lowther WT, Pemble CWt. Fatty acid synthase inhibitors: new directions for oncology. Expert Opin Investig Drugs 2007; 16: 1817-29.

29 Mashima T, Seimiya H, Tsuruo T. De novo fatty-acid synthesis and related pathways as molecular targets for cancer therapy. Br J Cancer 2009; 100: 1369-72.

30 Lupu R, Menendez JA. Targeting fatty acid synthase in breast and endometrial cancer: An alternative to selective estrogen receptor modulators? Endocrinology 2006; 147: 4056-66.

31 Yang YA, Han WF, Morin PJ, Chrest FJ, Pizer ES. Activation of fatty acid synthesis during neoplastic transformation: role of mitogen-activated protein kinase and phosphatidylinositol 3-kinase. Exp Cell Res 2002; 279: 80-90.

32 Menendez JA, Colomer R, Lupu R. Why does tumor-associated fatty acid synthase (oncogenic antigen-519) ignore dietary fatty acids? Med Hypotheses 2005; 64: 342-9.

33 Menna C, Olivieri F, Catalano A, Procopio A. Lipoxygenase inhibitors for cancer prevention: promises and risks. Curr Pharm Des 2010; 16: 725-33.

34 Avis I, Hong SH, Martinez A, Moody T, Choi YH, Trepel J, et al. Fivelipoxygenase inhibitors can mediate apoptosis in human breast cancer cell lines through complex eicosanoid interactions. FASEB J 2001; 15: 2007-9.

35 Wei N, Wang B, Zhang QY, Mi MT, Zhu JD, Yu XP, et al. Effects of different dietary fatty acids on the fatty acid compositions and the expression of lipid metabolic-related genes in mammary tumor tissues of rats. Nutr Cancer 2008; 60: 810-25.

36 Pidgeon GP, Lysaght J, Krishnamoorthy S, Reynolds JV, O‘Byrne K, Nie $D$, et al. Lipoxygenase metabolism: roles in tumor progression and survival. Cancer Metastasis Rev 2007; 26: 503-24.

37 Steinmetz R, Wagoner HA, Zeng P, Hammond JR, Hannon TS, Meyers $\mathrm{JL}$, et al. Mechanisms regulating the constitutive activation of the extracellular signal-regulated kinase (ERK) signaling pathway in ovarian cancer and the effect of ribonucleic acid interference for ERK1/2 on cancer cell proliferation. Mol Endocrinol 2004; 18: 2570-82.

38 Bachi AL, Kim FJ, Nonogaki S, Carneiro CR, Lopes JD, Jasiulionis MG, et al. Leukotriene B4 creates a favorable microenvironment for murine melanoma growth. Mol Cancer Res 2009; 7: 1417-24.

39 Shan C, Xu F, Zhang S, You J, You X, Qiu L, et al. Hepatitis B virus X protein promotes liver cell proliferation via a positive cascade loop involving arachidonic acid metabolism and p-ERK1/2. Cell Res 2010; 20: $563-75$. 